\title{
ASYMPTOTICS FOR DISCRETE WEIGHTED MINIMAL RIESZ ENERGY PROBLEMS ON RECTIFIABLE SETS
}

\author{
S. V. BORODACHOV, D. P. HARDIN, AND E. B. SAFF
}

\begin{abstract}
Given a closed $d$-rectifiable set $A$ embedded in Euclidean space, we investigate minimal weighted Riesz energy points on $A$; that is, $N$ points constrained to $A$ and interacting via the weighted power law potential $V=$ $w(x, y)|x-y|^{-s}$, where $s>0$ is a fixed parameter and $w$ is an admissible weight. (In the unweighted case $(w \equiv 1)$ such points for $N$ fixed tend to the solution of the best-packing problem on $A$ as the parameter $s \rightarrow \infty$.) Our main results concern the asymptotic behavior as $N \rightarrow \infty$ of the minimal energies as well as the corresponding equilibrium configurations. Given a distribution $\rho(x)$ with respect to $d$-dimensional Hausdorff measure on $A$, our results provide a method for generating $N$-point configurations on $A$ that are "well-separated" and have asymptotic distribution $\rho(x)$ as $N \rightarrow \infty$.
\end{abstract}

\section{INTRODUCTION}

Points on a compact set $A$ that minimize certain energy functions often have desirable properties that reflect special features of $A$. For $A=S^{2}$, the unit sphere in $\mathbb{R}^{3}$, the determination of minimal Coulomb energy points is the classic problem of Thomson [20,5]. Other energy functions on higher dimensional spheres give rise to equilibrium points that are useful for a variety of applications including coding theory [6], cubature formulas [21], and the generation of finite normalized tight frames [3]. In this paper, we shall consider a generalized Thomson problem, namely minimal energy points for weighted Riesz potentials on rectifiable sets. Our focus is on the hypersingular case when short range interaction between points is the dominant effect. Such energy functions are not treatable with classical potential theoretic methods, and so require different techniques of analysis.

Let $A$ be a compact set in $\mathbb{R}^{d^{\prime}}$ whose $d$-dimensional Hausdorff measure, $\mathcal{H}_{d}(A)$, is finite. For a collection of $N(\geq 2)$ distinct points $\omega_{N}:=\left\{x_{1}, \ldots, x_{N}\right\} \subset A$, a non-negative weight function $w$ on $A \times A$ (we shall specify additional conditions on

Received by the editors February 10, 2006.

2000 Mathematics Subject Classification. Primary 11K41, 70F10, 28A78; Secondary 78A30, $52 \mathrm{~A} 40$.

Key words and phrases. Minimal discrete Riesz energy, best-packing, Hausdorff measure, rectifiable sets, non-uniform distribution of points, power law potential, separation radius.

The research of the first author was conducted as a graduate student under the supervision of E.B. Saff and D. P. Hardin at Vanderbilt University.

The research of the second author was supported, in part, by the U. S. National Science Foundation under grants DMS-0505756 and DMS-0532154.

The research of the third author was supported, in part, by the U. S. National Science Foundation under grant DMS-0532154. 
$w$ shortly), and $s>0$, the weighted Riesz s-energy of $\omega_{N}$ is defined by

$$
E_{s}^{w}\left(\omega_{N}\right):=\sum_{1 \leq i \neq j \leq N} \frac{w\left(x_{i}, x_{j}\right)}{\left|x_{i}-x_{j}\right|^{s}}=\sum_{i=1}^{N} \sum_{\substack{j=1 \\ j \neq i}}^{N} \frac{w\left(x_{i}, x_{j}\right)}{\left|x_{i}-x_{j}\right|^{s}},
$$

while the $N$-point weighted Riesz s-energy of $A$ is defined by

$$
\mathcal{E}_{s}^{w}(A, N):=\inf \left\{E_{s}^{w}\left(\omega_{N}\right): \omega_{N} \subset A,\left|\omega_{N}\right|=N\right\},
$$

where $|X|$ denotes the cardinality of a set $X$. Since, for the weight $\widetilde{w}(x, y):=$ $(w(x, y)+w(y, x)) / 2$, we have

$$
E_{s}^{w}\left(\omega_{N}\right)=E_{s}^{\widetilde{w}}\left(\omega_{N}\right)=2 \sum_{1 \leq i<j \leq N} \frac{\widetilde{w}\left(x_{i}, x_{j}\right)}{\left|x_{i}-x_{j}\right|^{s}},
$$

we shall assume, without loss of generality, throughout this paper that $w$ is symmetric, i.e., $w(x, y)=w(y, x)$ for $x, y \in A$. We call $w: A \times A \rightarrow[0, \infty]$ a $C P D$-weight function on $A \times A$ if

(a) $w$ is continuous (as a function on $A \times A$ ) at $\mathcal{H}_{d}$-almost every point of the diagonal $D(A):=\{(x, x): x \in A\}$,

(b) there is some neighborhood $G$ of $D(A)$ (relative to $A \times A$ ) such that $\inf _{G} w>0$, and

(c) $w$ is bounded on any closed subset $B \subset A \times A$ such that $B \cap D(A)=\emptyset$.

Here CPD stands for (almost) continuous and positive on the diagonal. In particular, conditions (a), (b), and (c) hold if $w$ is bounded on $A \times A$ and continuous and positive at every point of the diagonal $D(A)$ (where continuity at a diagonal point $\left(x_{0}, x_{0}\right)$ is meant in the sense of limits taken on $\left.A \times A\right)$.

If $w \equiv 1$ on $A \times A$ (which we refer to as the unweighted case), we write $E_{s}\left(\omega_{N}\right)$ and $\mathcal{E}_{s}(A, N)$ for $E_{s}^{w}\left(\omega_{N}\right)$ and $\mathcal{E}_{s}^{w}(A, N)$, respectively. For the trivial cases $N=0$ or 1 we put $E_{s}\left(\omega_{N}\right)=\mathcal{E}_{s}(A, N)=E_{s}^{w}\left(\omega_{N}\right)=\mathcal{E}_{s}^{w}(A, N)=0$.

We are interested in the geometrical properties of optimal $s$-energy $N$-point configurations for a set $A$; that is, sets $\omega_{N}$ for which the infimum in (1) is attained. Indeed, these configurations are useful in statistical sampling, weighted quadrature, and computer-aided geometric design where the selection of a "good" finite (but possibly large) collection of points is required to represent a set or manifold $A$. Since the exact determination of optimal configurations seems, except in a handful of cases (cf. [23, 14, 1, 15, 2, 9]), beyond the realm of possibility, our focus is on the asymptotics of such configurations. Specifically, we consider the following questions:

(i) What is the asymptotic behavior of the quantity $\mathcal{E}_{s}^{w}(A, N)$ as $N$ gets large?

(ii) How are optimal point configurations distributed as $N \rightarrow \infty$ ?

(iii) What estimates can be given for the minimal pairwise distance between points in optimal configurations for large $N$ ?

In the unweighted case, much is known regarding these questions. In particular, when $s<\operatorname{dim} A$ (the Hausdorff dimension of $A$ ), the limit distribution of optimal $N$-point configurations is given by the equilibrium measure $\lambda_{s, A}$ that minimizes the continuous energy integral

$$
I_{s}(\mu):=\iint_{A \times A} \frac{1}{|x-y|^{s}} d \mu(x) d \mu(y)
$$


over the class $\mathcal{M}(A)$ of (Radon) probability measures $\mu$ supported on $A$. In addition, the asymptotic order of the Riesz $s$-energy is $N^{2}$; more precisely we have $\mathcal{E}_{s}(A, N) / N^{2} \rightarrow I_{s}\left(\lambda_{s, A}\right)$ as $N \rightarrow \infty$ (cf. [18, Section II.3.12]). In the case when $A=S^{d}$, the unit sphere in $\mathbb{R}^{d+1}$, the equilibrium measure is simply the normalized surface area measure.

If $s \geq \operatorname{dim} A$, then $I_{s}(\mu)=\infty$ for every $\mu \in \mathcal{M}(A)$, and potential theoretic methods cannot be used. However, by using techniques from geometric measure theory, it was recently shown in [12] that when $A$ is a $d$-rectifiable manifold of positive $d$-dimensional Hausdorff measure and $s \geq d$, optimal $N$-point configurations are uniformly distributed (as $N \rightarrow \infty$ ) on $A$ with respect to $d$-dimensional Hausdorff measure restricted to $A$. The assertion for the case $s=d$ further requires that $A$ be a subset of a $C^{1}$ manifold (see Theorem A in Section 1.1).

Our motivation for considering the weighted minimal Riesz energy problem is for the purpose of obtaining point sets that are distributed according to a specified non-uniform density such as might be used as nodes for weighted integration or in computer modeling of surfaces where more points are needed in regions with higher curvature. In this paper we shall show that for a compact $d$-rectifiable set $A$ having positive $d$-dimensional Hausdorff measure, $N$-point configurations for $A$ minimizing the weighted Riesz $s$-energy are distributed asymptotically with density proportional to $(w(x, x))^{-d / s}$ provided $s \geq d$. (This continues to be true even when $w$ has a finite number of zeros on the diagonal, provided their order is less than s.) As a consequence (cf. Corollary 2), given an appropriate distribution $\rho$, one can utilize minimal weighted energy points to generate a sequence of $N$-point configurations that are "well-separated" and have asymptotic distribution $\rho$. Even in the unweighted case, our results extend those of [12] obtained for the class of $d$-rectifiable manifolds to the more general class of $d$-rectifiable sets.

For the remainder of this introduction we provide the necessary notation and discuss known results. Section 2 is devoted to the statements of the main results of this paper. The detailed proofs of these main results, which utilize the basic lemmas described in Section 3, are provided in Sections 4, 5 and 6.

1.1. Notation and previous results. It is helpful to keep in mind that minimal discrete $s$-energy problems can be considered as a bridge between logarithmic energy problems and best-packing ones. Indeed, in the unweighted case $(w(x, y) \equiv 1)$ when $s \rightarrow 0$ and $N$ is fixed, the minimal energy problem turns into the problem for the logarithmic potential energy

$$
\sum_{1 \leq i \neq j \leq N} \log \frac{1}{\left|x_{i}-x_{j}\right|},
$$

which is minimized over all $N$-point configurations $\left\{x_{1}, \ldots, x_{N}\right\} \subset A$. This problem is equivalent to the maximization of the product

$$
\prod_{1 \leq i \neq j \leq N}\left|x_{i}-x_{j}\right|
$$

For planar sets, such optimal points are known as Fekete points. (For the case when $A=S^{2}$, the polynomial time generation of "nearly optimal" points for the logarithmic energy is the focus of one of S. Smale's "problems for the next century"; see [22].) 
On the other hand, when $s \rightarrow \infty$, and $N$ is fixed in the unweighted case, we get the best-packing problem (cf. [11], [6]); i.e., the problem of finding $N$-point configurations $\omega_{N} \subset A$ with the largest separation radius:

$$
\delta\left(\omega_{N}\right):=\min _{1 \leq i \neq j \leq N}\left|x_{i}-x_{j}\right| .
$$

In this paper we will consider the case $s \geq \operatorname{dim} A$. Let $\mathcal{L}_{d^{\prime}}$ be the Lebesgue measure in $\mathbb{R}^{d^{\prime}}$ and $\mathcal{H}_{d}$ be the $d$-dimensional Hausdorff measure in $\mathbb{R}^{d^{\prime}}$ normalized so that its restriction to $\mathbb{R}^{d} \subset \mathbb{R}^{d^{\prime}}$ is $\mathcal{L}_{d}$. Denote by $B_{d^{\prime}}\left(x_{0}, r\right)$ the open ball in $\mathbb{R}^{d^{\prime}}$ centered at the point $x_{0}$ with radius $r>0$ and set

$$
\beta_{d}:=\mathcal{L}_{d}\left(B_{d}(0,1)\right)=\frac{2 \pi^{d / 2}}{d \Gamma(d / 2)} .
$$

Given sequences $\left\{a_{N}\right\}_{N=1}^{\infty}$ and $\left\{b_{N}\right\}_{N=1}^{\infty}$ of positive numbers, we will write $a_{N} \sim b_{N}, N \rightarrow \infty$, if $\lim _{N \rightarrow \infty} a_{N} / b_{N}=1$.

Regarding questions (i) and (ii) concerning the asymptotics of minimal energy and of optimal configurations, A.B.J. Kuijlaars and E.B. Saff [16] proved that, for the unit sphere $S^{d}$,

$$
\mathcal{E}_{d}\left(S^{d}, N\right) \sim \frac{\beta_{d}}{\mathcal{H}_{d}\left(S^{d}\right)} N^{2} \log N, \quad N \rightarrow \infty,
$$

and it is known that the distribution of the minimal energy points is asymptotically uniform in this case. For one-dimensional rectifiable curves in $\mathbb{R}^{d^{\prime}}$, the paper by A. Martinez-Finkelshtein et al. [19] provides answers in the unweighted case to questions (i) and (ii), as well as question (iii) concerning separation of optimal points on regular Jordan arcs or curves.

Question (iii) in the unweighted case has also been considered for several other special cases. B.E.J. Dahlberg [7] proved that if $A=S^{d}$ and $s=d-1, d \geq 2$, or $A \subset \mathbb{R}^{3}$ is a smooth surface and $s=1, d=2$, there is a constant $C>0$ such that for every $s$-optimal collection $\omega_{N}^{*} \subset A$ with $N$ points

$$
\delta\left(\omega_{N}^{*}\right) \geq C N^{-1 / d} .
$$

In [16] it was shown that (4) holds for $A=S^{d}$ when $s>d$.

In [12] and [13], questions (i), (ii), and (iii) were addressed for a more general class of sets $A$ which we now describe. First recall that a mapping $\phi: T \rightarrow \mathbb{R}^{d^{\prime}}$, $T \subset \mathbb{R}^{d}$, is said to be a Lipschitz mapping on $T$ if there is some constant $\lambda$ such that

$$
|\phi(x)-\phi(y)| \leq \lambda|x-y| \quad \text { for } x, y \in T,
$$

and that $\phi$ is said to be a bi-Lipschitz mapping on $T$ (with constant $\lambda$ ) if

$$
(1 / \lambda)|x-y| \leq|\phi(x)-\phi(y)| \leq \lambda|x-y| \quad \text { for } x, y \in T .
$$

Following [12], we say that a set $A \subset \mathbb{R}^{d^{\prime}}$ is a $d$-rectifiable manifold if it is a compact subset of a finite union of bi-Lipschitz images of open sets in $\mathbb{R}^{d}$.

We further recall that if $A \subset \mathbb{R}^{d^{\prime}}$ is compact and $\nu$ and $\left\{\nu_{N}\right\}_{N=1}^{\infty}$ are Borel probability measures on $A$, then the sequence $\nu_{N}$ converges weak-star to $\nu$ (and we write $\nu_{N} \stackrel{*}{\longrightarrow} \nu$ ) if for any function $f$ continuous on $A$, we have

$$
\lim _{N \rightarrow \infty} \int_{A} f d \nu_{N}=\int_{A} f d \nu
$$

Denote by $\delta_{x}$ the atomic probability measure in $\mathbb{R}^{d^{\prime}}$ centered at the point $x \in \mathbb{R}^{d^{\prime}}$. 
For future reference and comparison we now state some of the main results from [12]. In the following theorems, the expression $1 / 0$ should be understood as $\infty$.

Theorem A. Let $A \subset \mathbb{R}^{d^{\prime}}$ be a compact d-rectifiable manifold and suppose $s>d$. Then

$$
\lim _{N \rightarrow \infty} \frac{\mathcal{E}_{s}(A, N)}{N^{1+s / d}}=\frac{C_{s, d}}{\mathcal{H}_{d}(A)^{s / d}},
$$

where $C_{s, d}$ is a positive constant independent of $A$.

Furthermore, if $\mathcal{H}_{d}(A)>0$, any asymptotically s-energy minimizing sequence of configurations $\widetilde{\omega}_{N}=\left\{x_{1}^{N}, \ldots, x_{N}^{N}\right\}, N=2,3, \ldots$, for $A$ is uniformly distributed with respect to $\mathcal{H}_{d}$; that is,

$$
\frac{1}{N} \sum_{k=1}^{N} \delta_{x_{k}^{N}} \stackrel{*}{\longrightarrow} \frac{\left.\mathcal{H}_{d}\right|_{A}}{\mathcal{H}_{d}(A)}, \quad N \rightarrow \infty .
$$

By asymptotically s-energy minimizing we mean

$$
E_{s}\left(\widetilde{\omega}_{N}\right) \sim \mathcal{E}_{s}(A, N), \quad N \rightarrow \infty .
$$

Remark. The constant $C_{s, d}$ appearing in (7) of Theorem A can be represented using the energy for the unit cube in $\mathbb{R}^{d}$ via formula (7):

$$
C_{s, d}=\lim _{N \rightarrow \infty} \frac{\mathcal{E}_{s}\left([0,1]^{d}, N\right)}{N^{1+s / d}}, s>d .
$$

For $d=1$ and $s>1$, it was shown in [19] that $C_{s, 1}=2 \zeta(s)$, where $\zeta(s)$ is the classical Riemann zeta function. However, for other values of $d$, the constant $C_{s, d}$ is as yet unknown. For the case $d=2$, it is a consequence of results in [16] that

$$
C_{s, 2} \leq(\sqrt{3} / 2)^{s / 2} \zeta_{L}(s)
$$

where $\zeta_{L}(s)$ is the zeta function for the planar triangular lattice $L$ consisting of points of the form $m(1,0)+n(1 / 2, \sqrt{3} / 2)$ for $m, n \in \mathbb{Z}$. It is conjectured in [16] that in fact equality holds in (9). Furthermore, it is shown in [4] that as $s \rightarrow \infty$

$$
\left(C_{s, 2}\right)^{2 / s} \rightarrow \sqrt{3} / 2,
$$

which is consistent with this conjecture.

When $0<\mathcal{H}_{d}(A)<\infty$ we observe that the minimum energy experiences a transition in order of growth; namely, as $s$ increases from values less than $d$ to values greater than $d$, the energy switches from order $N^{2}$ to order $N^{1+s / d}$ as $N \rightarrow \infty$. As the following theorem from [12] describes, at the transition value $s=d$, the order of growth is $N^{2} \log N$. In the proof of this fact, a more delicate analysis was utilized that required an additional regularity assumption on $A .^{1}$

Theorem B. Let $A$ be a compact subset of a d-dimensional $C^{1}$-manifold in $\mathbb{R}^{d^{\prime}}$. Then

$$
\lim _{N \rightarrow \infty} \frac{\mathcal{E}_{d}(A, N)}{N^{2} \log N}=\frac{\beta_{d}}{\mathcal{H}_{d}(A)},
$$

where $\beta_{d}$ is the volume of the d-dimensional unit ball as defined in (3).

\footnotetext{
${ }^{1}$ At this writing it is not known whether this requirement is necessary.
} 
Furthermore, if $\mathcal{H}_{d}(A)>0$, any asymptotically d-energy minimizing sequence of configurations $\widetilde{\omega}_{N}=\left\{x_{1}^{N}, \ldots, x_{N}^{N}\right\}, N=2,3, \ldots$, for $A$ is uniformly distributed with respect to $\mathcal{H}_{d}$; that is, (8) holds.

Regarding separation results, the following was shown in [12]. If $A \subset \mathbb{R}^{d^{\prime}}$ is a bi-Lipschitz image of a compact set from $\mathbb{R}^{d}$ of positive Lebesgue measure, then for every $s \geq d$ there is a constant $c_{s}>0$ such that

$$
\delta\left(\omega_{N}^{*}\right) \geq \begin{cases}c_{s} N^{-1 / d}, & s>d, \\ c_{d}(N \log N)^{-1 / d}, & s=d,\end{cases}
$$

for every $s$-optimal $N$-point configuration $\omega_{N}^{*}$ on $A$. This result was extended by S. Damelin and V. Maymeskul in [8] to a finite union of bi-Lipschitz images of compact sets from $\mathbb{R}^{d}$.

\section{MAin RESUlts}

In this paper we extend Theorem $\mathrm{A}$ to the class of $d$-rectifiable sets, where, by a $d$-rectifiable set $A \subset \mathbb{R}^{d^{\prime}}$, we mean the image of a bounded set in $\mathbb{R}^{d}$ under a Lipschitz mapping (cf. [10]). Consequently, we relax the bi-Lipschitz condition in Theorem A. Furthermore, both Theorems A and B are extended to the case of weighted energy, and separation estimates for optimal configurations are obtained even when the Hausdorff dimension of the compact set is not necessarily an integer.

Theorem 1. Suppose $A \subset \mathbb{R}^{d^{\prime}}$ is a closed d-rectifiable set. Then, for $s>d$,

$$
\lim _{N \rightarrow \infty} \frac{\mathcal{E}_{s}(A, N)}{N^{1+s / d}}=\frac{C_{s, d}}{\mathcal{H}_{d}(A)^{s / d}},
$$

where $C_{s, d}$ is the same constant as in Theorem $A$.

Remark. As can be seen from the proof in Section 3, Theorem 1 is valid for a possibly more general class of sets, namely $\left(\mathcal{H}_{d}, d\right)$-rectifiable sets whose d-dimensional Hausdorff measure equals its d-dimensional Minkowski content (see Section 3 for definitions).

If $A$ is a compact set in $\mathbb{R}^{d^{\prime}}$ and $w$ is a CPD-weight function on $A \times A$, then for $s \geq d$ we define the weighted Hausdorff measure $\mathcal{H}_{d}^{s, w}$ on Borel sets $B \subset A$ by

$$
\mathcal{H}_{d}^{s, w}(B):=\int_{B}(w(x, x))^{-d / s} d \mathcal{H}_{d}(x)
$$

and its normalized form by

$$
h_{d}^{s, w}(B):=\mathcal{H}_{d}^{s, w}(B) / \mathcal{H}_{d}^{s, w}(A) .
$$

We say that a sequence $\left\{\widetilde{\omega}_{N}\right\}_{N=1}^{\infty}$ of $N$-point configurations in $A$ is asymptotically $(w, s)$-energy minimizing for $A$ if

$$
E_{s}^{w}\left(\widetilde{\omega}_{N}\right) \sim \mathcal{E}_{s}^{w}(A, N), \quad N \rightarrow \infty .
$$

The main results of this paper include the following generalizations of Theorems A and B. In their proofs, Lemma 6 in Section 4 plays a crucial role. 
Theorem 2. Let $A \subset \mathbb{R}^{d^{\prime}}$ be a closed d-rectifiable set. Suppose $s>d$ and that $w$ is a $C P D$-weight function on $A \times A$. Then

$$
\lim _{N \rightarrow \infty} \frac{\mathcal{E}_{s}^{w}(A, N)}{N^{1+s / d}}=\frac{C_{s, d}}{\left[\mathcal{H}_{d}^{s, w}(A)\right]^{s / d}},
$$

where $C_{s, d}$ is the same constant as in Theorem $\mathrm{A}$.

Furthermore, if $\mathcal{H}_{d}(A)>0$, any asymptotically $(w, s)$-energy minimizing sequence of configurations $\widetilde{\omega}_{N}=\left\{x_{1}^{N}, \ldots, x_{N}^{N}\right\}, N=2,3, \ldots$, for $A$ is uniformly distributed with respect to $\mathcal{H}_{d}^{s, w}$; that is,

$$
\frac{1}{N} \sum_{k=1}^{N} \delta_{x_{k}^{N}} \stackrel{*}{\longrightarrow} h_{d}^{s, w}, \quad N \rightarrow \infty .
$$

Theorem 3. Let $A$ be a compact subset of a d-dimensional $C^{1}$-manifold in $\mathbb{R}^{d^{\prime}}$ and suppose $w$ is a CPD-weight function on $A \times A$. Then

$$
\lim _{N \rightarrow \infty} \frac{\mathcal{E}_{d}^{w}(A, N)}{N^{2} \log N}=\frac{\beta_{d}}{\mathcal{H}_{d}^{d, w}(A)} .
$$

Furthermore, if $\mathcal{H}_{d}(A)>0$, any asymptotically $(w, d)$-energy minimizing sequence of configurations $\widetilde{\omega}_{N}=\left\{x_{1}^{N}, \ldots, x_{N}^{N}\right\}, N=2,3, \ldots$, for $A$ is uniformly distributed with respect to $\mathcal{H}_{d}^{d, w}$; that is, (15) holds with $s=d$.

Remarks. In the case $\mathcal{H}_{d}(A)=0$, the right-hand sides of (14) and (16) are understood to be infinity.

Next we obtain estimates for the separation radius of optimal configurations on sets of arbitrary Hausdorff dimension $\alpha$. We remark that the normalization for the Hausdorff measure $\mathcal{H}_{\alpha}$ plays no essential role here.

Theorem 4. Let $0<\alpha \leq d^{\prime}$. Suppose $A \subset \mathbf{R}^{d^{\prime}}$ is a compact set with $\mathcal{H}_{\alpha}(A)>0$ and let $w$ be a CPD-weight function that is bounded and lower semi-continuous on $A \times A$. Then, for every $s \geq \alpha$ there is a constant $c_{s}=c_{s}(A, w, \alpha)>0$ such that any $(w, s)$-energy minimizing configuration $\omega_{N}^{*}:=\left\{x_{1, N}, \ldots, x_{N, N}\right\}$ on $A$ satisfies the inequality

$$
\delta\left(\omega_{N}^{*}\right)=\min _{1 \leq i \neq j \leq N}\left|x_{i, N}-x_{j, N}\right| \geq\left\{\begin{array}{l}
c_{s} N^{-1 / \alpha}, \quad s>\alpha \\
c_{\alpha}(N \log N)^{-1 / \alpha}, \quad s=\alpha, \quad N \geq 2 .
\end{array}\right.
$$

As a consequence of the proof of Theorem 4 we establish the following estimates. Let

$$
\mathcal{H}_{\alpha}^{\infty}(A):=\inf \left\{\sum_{i}\left(\operatorname{diam} G_{i}\right)^{\alpha}: A \subset \bigcup_{i} G_{i}\right\}
$$

Corollary 1. Under the assumptions of Theorem 4, for $N \geq 2$,

$$
\mathcal{E}_{s}^{w}(A, N) \leq\left\{\begin{array}{l}
M_{s, \alpha}\|w\|_{A \times A} \mathcal{H}_{\alpha}^{\infty}(A)^{-s / \alpha} N^{1+s / \alpha}, \quad s>\alpha, \\
M_{\alpha} N^{2} \log N, \quad s=\alpha,
\end{array}\right.
$$

where the constant $M_{s, \alpha}>0$ is independent of $A, w$ and $N$, and the constant $M_{\alpha}$ is independent of $N$. 
In order to obtain a finite collection of points distributed with a given density $\rho(x)$ on a $d$-rectifiable set $A$, we can take any $s>d$ and the weight

$$
w(x, y):=(\rho(x) \rho(y)+|x-y|)^{-s / 2 d},
$$

where the term $|x-y|$ is included to ensure that $w$ is locally bounded off $D(A)$. By Theorems 2 and 3 any asymptotically $(w, s)$-energy minimizing sequence of $N$-point configurations will converge to the required distribution as $N \rightarrow \infty$. We thus obtain

Corollary 2. Let $A \subset \mathbb{R}^{d^{\prime}}$ be a closed d-rectifiable set with $\mathcal{H}_{d}(A)>0$. Suppose $\rho$ is a bounded probability density on $A$ (with respect to $\mathcal{H}_{d}$ ) that is continuous $\mathcal{H}_{d}$-almost everywhere on $A$. Then, for $s>d$ and $w$ given by (18), the normalized counting measures for any asymptotically $(w, s)$-energy minimizing sequence of configurations $\omega_{N}$ converge weak ${ }^{*}$ (as $N \rightarrow \infty$ ) to $\rho d \mathcal{H}_{d}$.

Furthermore, if $\inf _{A} \rho>0$ and $\rho$ is upper semi-continuous, then any $(w, s)$ energy minimizing sequence of configurations $\omega_{N}$ is well-separated in the sense of Theorem 4 with $\alpha=d$.

Remark. The first part of Corollary 2 holds for $s=d$ when $A$ is contained in a $C^{1}$ d-dimensional manifold.

Finally, we consider weight functions with isolated zeros. For $t>0$, we say that a function $w: A \times A \rightarrow \mathbb{R}$ has a zero at $(a, a) \in D(A)$ of order at most $t$ if there are positive constants $C$ and $\delta$ such that

$$
w(x, y) \geq C|x-a|^{t} \quad\left(x, y \in A \cap B_{d^{\prime}}(a, \delta)\right) .
$$

If $w$ has a zero $a \in A$ whose order is too large, then $a$ may act as an attractive "sink" with $\mathcal{E}_{s}^{w}(A, N)=0$. For example, let $A$ be the closed unit ball in $\mathbb{R}^{d}$, $w(x, y)=|x|^{t}+|y|^{t}$ for $x, y \in A$ with $t>s>d$. If $\omega_{N}=\left\{x_{1}, \ldots, x_{N}\right\}$ is a configuration of $N$ points in $A$, then $E_{s}^{w}\left(\gamma \omega_{N}\right)=\gamma^{t-s} E_{s}^{w}\left(\omega_{N}\right)$ for any $0<\gamma<1$. Taking $\gamma \rightarrow 0$ shows that $\mathcal{E}_{s}^{w}(A, N)=0$.

A closed set $A \subset \mathbb{R}^{d^{\prime}}$ is $\alpha$-regular at $a \in A$ if there are positive constants $C_{0}$ and $\delta$ such that

$$
\left(C_{0}\right)^{-1} r^{\alpha} \leq \mathcal{H}_{\alpha}\left(A \cap B_{d^{\prime}}(x, r)\right) \leq C_{0} r^{\alpha}
$$

for all $x \in A \cap B_{d^{\prime}}(a, \delta)$ and $0<r<\delta$.

Theorem 5. Let $A \subset \mathbb{R}^{d^{\prime}}$ be a closed d-rectifiable set and $s>d$. Suppose $A$ is $\alpha_{i}$-regular with $\alpha_{i} \leq d$ at $a_{i}, i=1, \ldots, n$, for a finite collection of points $a_{1}, \ldots, a_{n}$ in $A$ and that $w: A \times A \rightarrow[0, \infty]$ is a CPD-weight function on $K \times K$ for any compact $K \subset A \backslash\left\{a_{1}, \ldots, a_{n}\right\}$. If $w$ has zeros of order at most $t<s$ at each $\left(a_{i}, a_{i}\right)$, then the conclusions of Theorem 2 hold.

Remark. The hypotheses of Theorem 5 imply that

$$
\mathcal{H}_{d}^{s, w}(A)=\int_{A}(w(x, x))^{-d / s} d \mathcal{H}_{d}(x)
$$

is finite and positive (see Section 6) and, hence, the same is true of the right-hand side of (14).

\section{LEMMAS}

In this section we prove several lemmas which are central to the proofs of our main theorems. 
3.1. Divide and conquer. In this subsection we provide two lemmas relating the minimal energy problem on $A=B \cup D$ to the minimal energy problems on $B$ and $D$, respectively.

In order to unify our computations for the cases $s>d$ and $s=d$, we define, for integers $N>1$,

$$
\tau_{s, d}(N):= \begin{cases}N^{1+s / d}, & s>d \\ N^{2} \log N, & s=d\end{cases}
$$

and set $\tau_{s, d}(N)=1$ for $N=0$ or 1 . For a set $A \subset \mathbb{R}^{d^{\prime}}$ and $s \geq d$, let

$$
\underline{g}_{s, d}^{w}(A):=\liminf _{N \rightarrow \infty} \frac{\mathcal{E}_{s}^{w}(A, N)}{\tau_{s, d}(N)}, \quad \bar{g}_{s, d}^{w}(A):=\limsup _{N \rightarrow \infty} \frac{\mathcal{E}_{s}^{w}(A, N)}{\tau_{s, d}(N)}
$$

and

$$
g_{s, d}^{w}(A):=\lim _{N \rightarrow \infty} \frac{\mathcal{E}_{s}^{w}(A, N)}{\tau_{s, d}(N)}
$$

if this limit exists (these quantities are allowed to be infinite). In the case $w(x, y) \equiv$ 1 , we use the notations $\underline{g}_{s, d}(A), \bar{g}_{s, d}(A)$ and $g_{s, d}(A)$, respectively.

Let $\operatorname{dist}(B, D):=\inf \{|x-y|: x \in B, y \in D\}$ denote the distance between sets $B, D \subset \mathbb{R}^{d^{\prime}}$. The following two lemmas extend Lemmas 3.2 and 3.3 from [12] to the weighted case. We remark that the following results hold when quantities are 0 or infinite using $0^{-d / s}=0^{-s / d}=\infty$ and $\infty^{-d / s}=\infty^{-s / d}=0$.

Lemma 1. Let $s \geq d>0$ and suppose that $B$ and $D$ are sets in $\mathbb{R}^{d^{\prime}}$ such that $\operatorname{dist}(B, D)>0$. Suppose $w:(B \cup D) \times(B \cup D) \rightarrow[0, \infty]$ is bounded on the subset $B \times D$. Then

$$
\bar{g}_{s, d}^{w}(B \cup D)^{-d / s} \geq \bar{g}_{s, d}^{w}(B)^{-d / s}+\bar{g}_{s, d}^{w}(D)^{-d / s} .
$$

Proof. Assume that $0<\bar{g}_{s, d}^{w}(B), \bar{g}_{s, d}^{w}(D)<\infty$. Define

$$
\alpha^{*}:=\frac{\bar{g}_{s, d}^{w}(D)^{d / s}}{\bar{g}_{s, d}^{w}(B)^{d / s}+\bar{g}_{s, d}^{w}(D)^{d / s}} .
$$

For $N \in \mathbb{N}$, let $N_{B}:=\left\lfloor\alpha^{*} N\right\rfloor$ (where $\lfloor x\rfloor$ denotes the greatest integer less than or equal to $x), N_{D}:=N-N_{B}$ and $\omega_{N}^{B} \subset B$ and $\omega_{N}^{D} \subset D$ be configurations of $N_{B}$ and $N_{D}$ points respectively such that $E_{s}^{w}\left(\omega_{N}^{B}\right)<\mathcal{E}_{s}^{w}\left(B, N_{B}\right)+1$ and $E_{s}^{w}\left(\omega_{N}^{D}\right)<$ $\mathcal{E}_{s}^{w}\left(D, N_{D}\right)+1$. Let $\gamma_{0}:=\operatorname{dist}(B, D)>0$. Then

$$
\begin{aligned}
\mathcal{E}_{s, d}^{w}(B \cup D, N) & \leq E_{s}^{w}\left(\omega_{N}^{B} \cup \omega_{N}^{D}\right) \\
& =E_{s}^{w}\left(\omega_{N}^{B}\right)+E_{s}^{w}\left(\omega_{N}^{D}\right)+2 \sum_{x \in \omega_{N}^{B}, y \in \omega_{N}^{D}} \frac{w(x, y)}{|x-y|^{s}} \\
& \leq \mathcal{E}_{s}^{w}\left(B, N_{B}\right)+\mathcal{E}_{s}^{w}\left(D, N_{D}\right)+2+2 \gamma_{0}^{-s} N^{2}\|w\|_{B \times D},
\end{aligned}
$$


where $\|w\|_{B \times D}$ denotes the supremum of $w$ over $B \times D$. Dividing by $\tau_{s, d}(N)$ and taking into account that $\tau_{s, d}\left(N_{B}\right) / \tau_{s, d}(N) \rightarrow\left(\alpha^{*}\right)^{1+s / d}$ as $N \rightarrow \infty$, we obtain

$$
\begin{aligned}
\bar{g}_{s, d}^{w}(B \cup D) & \leq \limsup _{N \rightarrow \infty} \frac{\mathcal{E}_{s}^{w}\left(B, N_{B}\right)}{\tau_{s, d}(N)}+\limsup _{N \rightarrow \infty} \frac{\mathcal{E}_{s}^{w}\left(D, N_{D}\right)}{\tau_{s, d}(N)} \\
& =\limsup _{N \rightarrow \infty} \frac{\mathcal{E}_{s}^{w}\left(B, N_{B}\right)}{\tau_{s, d}\left(N_{B}\right)} \cdot \frac{\tau_{s, d}\left(N_{B}\right)}{\tau_{s, d}(N)}+\limsup _{N \rightarrow \infty} \frac{\mathcal{E}_{s}^{w}\left(D, N_{D}\right)}{\tau_{s, d}\left(N_{D}\right)} \cdot \frac{\tau_{s, d}\left(N_{D}\right)}{\tau_{s, d}(N)} \\
& \leq \bar{g}_{s, d}^{w}(B) \cdot\left(\alpha^{*}\right)^{1+s / d}+\bar{g}_{s, d}^{w}(D) \cdot\left(1-\alpha^{*}\right)^{1+s / d} \\
& =\left(\bar{g}_{s, d}^{w}(B)^{-d / s}+\bar{g}_{s, d}^{w}(D)^{-d / s}\right)^{-s / d} .
\end{aligned}
$$

The remaining cases when $\bar{g}_{s, d}^{w}(B)$ or $\bar{g}_{s, d}^{w}(D)$ are 0 or $\infty$ easily follow from the monotonicity of $\bar{g}_{s, d}^{w}$.

The following statement in particular shows sub-additivity of $\underline{g}_{s, d}^{w}(\cdot)^{-d / s}$ as well as provides a result relating asymptotics of energy to the limiting distribution of corresponding configurations.

Lemma 2. Let $s \geq d>0$ and $B, D \subset \mathbb{R}^{d^{\prime}}$. Suppose $w:(B \cup D) \times(B \cup D) \rightarrow[0, \infty]$. Then

$$
\underline{g}_{s, d}^{w}(B \cup D)^{-d / s} \leq \underline{g}_{s, d}^{w}(B)^{-d / s}+\underline{g}_{s, d}^{w}(D)^{-d / s} .
$$

Furthermore, if $\underline{g}_{s, d}^{w}(B), \underline{g}_{s, d}^{w}(D)>0$ and at least one of these quantities is finite, then for any infinite subset $\mathcal{N}$ of $\mathbb{N}$ and sequence $\left\{\widetilde{\omega}_{N}\right\}_{N \in \mathcal{N}}$ of $N$-point configurations in $B \cup D$ such that

$$
\lim _{\mathcal{N} \ni N \rightarrow \infty} \frac{E_{s}^{w}\left(\widetilde{\omega}_{N}\right)}{\tau_{s, d}(N)}=\left(\underline{g}_{s, d}^{w}(B)^{-d / s}+\underline{g}_{s, d}^{w}(D)^{-d / s}\right)^{-s / d}
$$

holds, we have

$$
\lim _{\mathcal{N} \ni N \rightarrow \infty} \frac{\left|\widetilde{\omega}_{N} \cap B\right|}{N}=\frac{\underline{g}_{s, d}^{w}(D)^{d / s}}{\underline{g}_{s, d}^{w}(B)^{d / s}+\underline{g}_{s, d}^{w}(D)^{d / s}} .
$$

In the case $\underline{g}_{s, d}^{w}(D)=\infty$ the right-hand side of relation (24) is understood to equal 1.

Proof. Assume that $0<\underline{g}_{s, d}^{w}(B), \underline{g}_{s, d}^{w}(D)<\infty$ (we leave other cases to the reader). Let an infinite subset $\mathcal{N}_{1} \subset \mathbb{N}$ and a sequence of point configurations $\left\{\omega_{N}\right\}_{N \in \mathcal{N}_{1}}$, $\omega_{N} \subset B \cup D$, be such that $\lim _{\mathcal{N}_{1} \ni N \rightarrow \infty}\left|\omega_{N} \cap B\right| / N=\alpha$, where $0 \leq \alpha \leq 1$. Set $N_{B}:=\left|\omega_{N} \cap B\right|$ and $N_{D}:=\left|\omega_{N} \backslash B\right|$. Then

$$
E_{s}^{w}\left(\omega_{N}\right) \geq E_{s}^{w}\left(\omega_{N} \cap B\right)+E_{s}^{w}\left(\omega_{N} \backslash B\right) \geq \mathcal{E}_{s}^{w}\left(B, N_{B}\right)+\mathcal{E}_{s}^{w}\left(D, N_{D}\right),
$$

and we have

$$
\begin{aligned}
& \liminf _{\mathcal{N}_{1} \ni N \rightarrow \infty} \frac{E_{s}^{w}\left(\omega_{N}\right)}{\tau_{s, d}(N)} \geq \liminf _{\mathcal{N}_{1} \ni N \rightarrow \infty} \frac{\mathcal{E}_{s}^{w}\left(B, N_{B}\right)}{\tau_{s, d}\left(N_{B}\right)} \cdot \frac{\tau_{s, d}\left(N_{B}\right)}{\tau_{s, d}(N)} \\
&+\liminf _{\mathcal{N}_{1} \ni N \rightarrow \infty} \frac{\mathcal{E}_{s}^{w}\left(D, N_{D}\right)}{\tau_{s, d}\left(N_{D}\right)} \cdot \frac{\tau_{s, d}\left(N_{D}\right)}{\tau_{s, d}(N)} \\
& \geq F(\alpha):=\underline{g}_{s, d}^{w}(B) \alpha^{1+s / d}+\underline{g}_{s, d}^{w}(D)(1-\alpha)^{1+s / d} .
\end{aligned}
$$


Let

$$
\widetilde{\alpha}:=\frac{\underline{g}_{s, d}^{w}(D)^{d / s}}{\underline{g}_{s, d}^{w}(B)^{d / s}+\underline{g}_{s, d}^{w}(D)^{d / s}},
$$

and $\left\{\widetilde{\omega}_{N}\right\}_{N \in \mathcal{N}}$ be any sequence of point sets satisfying (23). If $\mathcal{N}_{2} \subset \mathcal{N}$ is any infinite subsequence such that the quantity $\left|\widetilde{\omega}_{N} \cap B\right| / N$ has a limit as $\mathcal{N}_{2} \ni N \rightarrow \infty$ (denote it by $\alpha_{1}$ ), then in the case $\underline{g}_{s, d}^{w}(B), \underline{g}_{s, d}^{w}(D)<\infty$ by $(23)$ and $(25)$ we have

$$
F(\widetilde{\alpha})=\lim _{\mathcal{N}_{2} \ni N \rightarrow \infty} \frac{E_{s}^{w}\left(\widetilde{\omega}_{N}\right)}{\tau_{s, d}(N)} \geq F\left(\alpha_{1}\right),
$$

where we used the fact that $F(\widetilde{\alpha})$ is the right-hand side of (23). It is not difficult to see that $\widetilde{\alpha}$ is the only minimum point of $F(t)$ on $[0,1]$. Hence $\alpha_{1}=\widetilde{\alpha}$, which proves (24).

Now let $\left\{\bar{\omega}_{N}\right\}_{N \in \mathcal{N}_{3}}$ be a sequence of $N$-point configurations in $B \cup D$ such that

$$
\underline{g}_{s, d}^{w}(B \cup D)=\lim _{\mathcal{N}_{3} \ni N \rightarrow \infty} \frac{E_{s}^{w}\left(\bar{\omega}_{N}\right)}{\tau_{s, d}(N)}
$$

$\left(\bar{\omega}_{N}\right.$ 's can be chosen for example so that $\left.E_{s}^{w}\left(\bar{\omega}_{N}\right)<\mathcal{E}_{s}^{w}(B \cup D, N)+1\right)$. If $\mathcal{N}_{4} \subset \mathcal{N}_{3}$ is such an infinite set that $\lim _{\mathcal{N}_{4} \ni N \rightarrow \infty}\left|\bar{\omega}_{N} \cap B\right| / N$ exists (denote it by $\alpha_{2}$ ), then by (25) we obtain

$$
\begin{aligned}
\underline{g}_{s, d}^{w}(B \cup D) & =\lim _{\mathcal{N}_{4} \ni N \rightarrow \infty} \frac{E_{s}^{w}\left(\bar{\omega}_{N}\right)}{\tau_{s, d}(N)} \geq F\left(\alpha_{2}\right) \\
& \geq F(\widetilde{\alpha})=\left(\underline{g}_{s, d}^{w}(B)^{-d / s}+\underline{g}_{s, d}^{w}(D)^{-d / s}\right)^{-s / d},
\end{aligned}
$$

which implies (22).

Remark. An immediate consequence of the non-weighted versions of Lemmas 1 and 2 is Corollary 3.4 in [12]. We take this opportunity to remark that the hypotheses of that corollary should require that $B$ not intersect the interior of $A$ rather than the assumption that the two sets have disjoint interiors. However, this corollary was only applied in the former setting in [12].

3.2. Lemmas from geometric measure theory. Recall that $\beta_{d}$ denotes the volume of the unit ball in $\mathbb{R}^{d}$. For convenience, we also define $\beta_{0}:=1$. For a set $W \subset \mathbb{R}^{d^{\prime}}$ and $h>0$, we let

$$
W(h):=\left\{x \in \mathbb{R}^{d^{\prime}}: \operatorname{dist}(x, W)<h\right\} .
$$

The upper and the lower Minkowski contents of the set $W$ are defined, respectively, by

$$
\overline{\mathcal{M}}_{d}(W):=\limsup _{r \rightarrow 0^{+}} \frac{\mathcal{L}_{d^{\prime}}[W(r)]}{\beta_{d^{\prime}-d} \cdot r^{d^{\prime}-d}} \quad \text { and } \quad \underline{\mathcal{M}}_{d}(W):=\liminf _{r \rightarrow 0^{+}} \frac{\mathcal{L}_{d^{\prime}}[W(r)]}{\beta_{d^{\prime}-d} \cdot r^{d^{\prime}-d}} .
$$

If the upper and the lower Minkowski contents of the set $W$ coincide, then this common value, denoted by $\mathcal{M}_{d}(W)$, is called the Minkowski content of $W$. We shall rely on the following property of closed $d$-rectifiable sets.

Lemma 3 (see [10, Theorem 3.2.39]). If $W \subset \mathbb{R}^{d^{\prime}}$ is a closed d-rectifiable set, then $\mathcal{M}_{d}(W)=\mathcal{H}_{d}(W)$.

We shall also need the following fundamental lemma from geometric measure theory. 
Lemma 4 (see [10, Lemma 3.2.18]). Let $W \subset \mathbb{R}^{d^{\prime}}$ be a d-rectifiable set. Then for every $\epsilon>0$ there exist compact sets $K_{1}, K_{2}, K_{3}, \ldots \subset \mathbb{R}^{d}$ and bi-Lipschitz mappings $\psi_{i}: K_{i} \rightarrow \mathbb{R}^{d^{\prime}}$ with constant $1+\epsilon, i=1,2,3, \ldots$, such that $\psi_{1}\left(K_{1}\right)$, $\psi_{2}\left(K_{2}\right), \psi_{3}\left(K_{3}\right), \ldots$ are disjoint subsets of $W$ with

$$
\mathcal{H}_{d}\left(W \backslash \bigcup_{i} \psi_{i}\left(K_{i}\right)\right)=0
$$

In fact, the above lemma holds for any set of finite $\mathcal{H}_{d}$-measure that, up to a set of $\mathcal{H}_{d}$-measure zero, is the countable union of $d$-rectifiable sets. Such sets are called $\left(\mathcal{H}_{d}, d\right)$-rectifiable (cf. [10]). However, Lemma 3 does not hold for this larger class.

3.3. Regularity Lemma. To get an estimate from below for $\underline{g}_{s, d}(A)$ we will need the following result.

Lemma 5. Let $s>d$ and suppose $A \subset \mathbb{R}^{d^{\prime}}$ is a compact set such that $\mathcal{M}_{d}(A)$ exists and is finite. Then for every $\epsilon \in(0,1)$ there is some $\delta>0$ such that for any compact set $K \subset A$ with $\underline{\mathcal{M}}_{d}(K)>\mathcal{M}_{d}(A)-\delta$ we have

$$
\underline{g}_{s, d}(A) \geq(1-\epsilon) \underline{g}_{s, d}(K) .
$$

Proof. The assertion of the lemma holds trivially if $\underline{g}_{s, d}(A)=\infty$. Hence, we assume $\underline{g}_{s, d}(A)<\infty$. Let $\mathcal{N} \subset \mathbb{N}$ be an infinite subset such that

$$
\lim _{\mathcal{N} \ni N \rightarrow \infty} \frac{\mathcal{E}_{s}(A, N)}{N^{1+s / d}}=\underline{g}_{s, d}(A) .
$$

Choose $0<\rho<1 / 2$ and set

$$
\delta:=\rho^{4 d} \quad \text { and } \quad h_{N}:=\frac{1}{3} \rho^{2} N^{-1 / d}, \quad N \in \mathcal{N} .
$$

(Later in the proof, $\rho$ and hence $\delta$, will be chosen sufficiently small.) Suppose $K$ is a compact subset of $A$ such that $\underline{\mathcal{M}}_{d}(K)>\mathcal{M}_{d}(A)-\delta$. Then there is some $N_{\delta} \in \mathbb{N}$ such that for any $N>N_{\delta}, N \in \mathcal{N}$, we have

$$
\frac{\mathcal{L}_{d^{\prime}}\left[A\left(h_{N}\right)\right]}{\beta_{d^{\prime}-d} h_{N}^{d^{\prime}-d}} \leq \mathcal{M}_{d}(A)+\delta \quad \text { and } \quad \frac{\mathcal{L}_{d^{\prime}}\left[K\left(h_{N}\right)\right]}{\beta_{d^{\prime}-d} h_{N}^{d^{\prime}-d}} \geq \mathcal{M}_{d}(A)-\delta .
$$

For $N \in \mathcal{N}$ with $N>N_{\delta}$, let $\omega_{N}^{*}:=\left\{x_{1, N}, \ldots, x_{N, N}\right\}$ be an $s$-energy minimizing $N$-point configuration on $A$. For $i=1, \ldots, N$, let $r_{i, N}:=\min _{j \neq i}\left|x_{j, N}-x_{i, N}\right|$ denote the distance from $x_{i, N}$ to its nearest neighbor in $\omega_{N}^{*}$. Further, we partition $\omega_{N}^{*}$ into a "well-separated" subset

$$
\omega_{N}^{1}:=\left\{x_{i, N} \in \omega_{N}^{*}: r_{i, N} \geq \rho N^{-1 / d}\right\},
$$

and its complement $\widetilde{\omega}_{N}^{1}:=\omega_{N}^{*} \backslash \omega_{N}^{1}$. We next show that $\omega_{N}^{1}$ has sufficiently many points. For $N \in \mathcal{N}$, we obtain

$$
\begin{aligned}
\mathcal{E}_{s}(A, N) & =E_{s}\left(\omega_{N}^{*}\right)=\sum_{i=1}^{N} \sum_{\substack{j=1 \\
j \neq i}}^{N} \frac{1}{\left|x_{i, N}-x_{j, N}\right|^{s}} \geq \sum_{i=1}^{N} \frac{1}{\left(r_{i, N}\right)^{s}} \\
& \geq \sum_{x_{i, N} \in \widetilde{\omega}_{N}^{1}} \frac{1}{\left(r_{i, N}\right)^{s}} \geq \sum_{x_{i, N} \in \widetilde{\omega}_{N}^{1}} \frac{1}{\left(\rho N^{-1 / d}\right)^{s}}=\left|\widetilde{\omega}_{N}^{1}\right| \rho^{-s} N^{s / d} .
\end{aligned}
$$


Let $k_{0}:=\underline{g}_{s, d}(A)+1$. There is $N_{1} \in \mathbb{N}$ such that for any $N>N_{1}, N \in \mathcal{N}$,

$$
\frac{\mathcal{E}_{s}(A, N)}{N^{1+s / d}}<k_{0}
$$

For the rest of the proof of this lemma, let $N \in \mathcal{N}$ be greater than $N_{2}:=$ $\max \left\{N_{1}, N_{\delta}\right\}$. Then,

$$
\frac{\left|\widetilde{\omega}_{N}^{1}\right|}{\rho^{s} N} \leq \frac{\mathcal{E}_{s}(A, N)}{N^{1+s / d}}<k_{0},
$$

and, hence, we have

$$
\left|\widetilde{\omega}_{N}^{1}\right|<k_{0} \rho^{s} N \text { and }\left|\omega_{N}^{1}\right|>\left(1-k_{0} \rho^{s}\right) N
$$

Recalling the definition of $h_{N}$ in $(27)$, we next consider

$$
\omega_{N}^{2}:=\omega_{N}^{1} \bigcap K\left(3 h_{N}\right), \quad \widetilde{\omega}_{N}^{2}:=\omega_{N}^{1} \backslash K\left(3 h_{N}\right),
$$

and show that the cardinality of $\omega_{N}^{2}$ is sufficiently large. From (28) we get

$$
\begin{aligned}
\mathcal{L}_{d^{\prime}}\left[A\left(h_{N}\right) \backslash\right. & \left.K\left(h_{N}\right)\right]=\mathcal{L}_{d^{\prime}}\left[A\left(h_{N}\right)\right]-\mathcal{L}_{d^{\prime}}\left[K\left(h_{N}\right)\right] \\
\leq & \left(\mathcal{M}_{d}(A)+\delta\right) \beta_{d^{\prime}-d} h_{N}^{d^{\prime}-d}-\left(\mathcal{M}_{d}(A)-\delta\right) \beta_{d^{\prime}-d} h_{N}^{d^{\prime}-d} \\
& =2 \beta_{d^{\prime}-d} \delta h_{N}^{d^{\prime}-d} .
\end{aligned}
$$

Note that

$$
F_{N}:=\bigcup_{x \in \widetilde{\omega}_{N}^{2}} B_{d^{\prime}}\left(x, h_{N}\right) \subset A\left(h_{N}\right) \backslash K\left(h_{N}\right)
$$

For any distinct points $x_{i, N}, x_{j, N} \in \widetilde{\omega}_{N}^{2}$ we have

$$
\left|x_{i, N}-x_{j, N}\right| \geq r_{i, N} \geq \rho N^{-1 / d}>\rho^{2} N^{-1 / d}=3 h_{N} .
$$

Hence, $B_{d^{\prime}}\left(x_{i, N}, h_{N}\right) \bigcap B_{d^{\prime}}\left(x_{j, N}, h_{N}\right)=\emptyset$. Then, using (30) and (31), we get

$$
\begin{aligned}
\left|\widetilde{\omega}_{N}^{2}\right| & =\left(\beta_{d^{\prime}} h_{N}^{d^{\prime}}\right)^{-1} \sum_{x \in \widetilde{\omega}_{N}^{2}} \mathcal{L}_{d^{\prime}}\left[B_{d^{\prime}}\left(x, h_{N}\right)\right]=\left(\beta_{d^{\prime}} h_{N}^{d^{\prime}}\right)^{-1} \mathcal{L}_{d^{\prime}}\left(F_{N}\right) \\
& \leq\left(\beta_{d^{\prime}} h_{N}^{d^{\prime}}\right)^{-1} \mathcal{L}_{d^{\prime}}\left[A\left(h_{N}\right) \backslash K\left(h_{N}\right)\right] \leq 2 \beta_{d^{\prime}-d} \beta_{d^{\prime}}^{-1} \delta h_{N}^{-d} .
\end{aligned}
$$

Hence, recalling from $(27)$ that $h_{N}:=\frac{1}{3} \rho^{2} N^{-1 / d}$, we have

$$
\left|\widetilde{\omega}_{N}^{2}\right| \leq 2 \cdot 3^{d} \beta_{d^{\prime}-d} \beta_{d^{\prime}}^{-1} \delta^{1 / 2} N .
$$

Let $\chi_{0}:=2 \cdot 3^{d} \beta_{d^{\prime}-d} \beta_{d^{\prime}}^{-1}$. Then, using (29) and (32), we have

$$
\left|\omega_{N}^{2}\right|=\left|\omega_{N}^{1}\right|-\left|\widetilde{\omega}_{N}^{2}\right| \geq\left(1-k_{0} \rho^{s}-\chi_{0} \delta^{1 / 2}\right) N
$$

Next, we choose a configuration $\omega_{N}^{K}$ of points in $K$ which is close to $\omega_{N}^{2}$ and has the same number of points and order of the minimal $s$-energy as $\omega_{N}^{2}$. For every $x_{i, N} \in \omega_{N}^{2}$ pick a point $y_{i, N} \in K$ such that $\left|x_{i, N}-y_{i, N}\right|<3 h_{N}=\rho^{2} N^{-1 / d}$ and let 
$\omega_{N}^{K}:=\left\{y_{i, N}: x_{i, N} \in \omega_{N}^{2}\right\}$. Since every point $x_{i, N} \in \omega_{N}^{2}$ lies in $\omega_{N}^{1}$, we have

$$
\left|x_{i, N}-y_{i, N}\right|<\rho^{2} N^{-1 / d} \leq \rho r_{i, N} \leq \rho\left|x_{i, N}-x_{j, N}\right|, \quad j \neq i .
$$

Then, if $x_{i, N} \neq x_{j, N}$ are points from $\omega_{N}^{2}$, we have

$$
\begin{aligned}
\left|y_{i, N}-y_{j, N}\right| & =\left|y_{i, N}-x_{i, N}+x_{i, N}-x_{j, N}+x_{j, N}-y_{j, N}\right| \\
& \geq\left|x_{i, N}-x_{j, N}\right|-\left|x_{i, N}-y_{i, N}\right|-\left|x_{j, N}-y_{j, N}\right| \\
& \geq\left|x_{i, N}-x_{j, N}\right|-2 \rho\left|x_{i, N}-x_{j, N}\right|=(1-2 \rho)\left|x_{i, N}-x_{j, N}\right| .
\end{aligned}
$$

Since $\rho \in(0,1 / 2)$, it follows that $\left|\omega_{N}^{K}\right|=\left|\omega_{N}^{2}\right|$ and

$$
\begin{aligned}
E_{s}\left(\omega_{N}^{*}\right) & =\sum_{x \neq y \in \omega_{N}^{*}} \frac{1}{|x-y|^{s}} \geq \sum_{x \neq y \in \omega_{N}^{2}} \frac{1}{|x-y|^{s}} \\
& \geq(1-2 \rho)^{s} \sum_{\substack{x \neq y \in \omega_{N}^{K} \\
|x-y|^{s}}} \frac{1}{\mid 1-2 \rho)^{s} E_{s}\left(\omega_{N}^{K}\right) .}
\end{aligned}
$$

Now suppose $\epsilon \in(0,1)$. We may choose $\delta>0$ sufficiently small (recall $\rho=$ $\left.\delta^{1 /(4 d)}\right)$ so that $(1-2 \rho)^{s}\left(1-k_{0} \rho^{s}-\chi_{0} \delta^{1 / 2}\right)^{1+s / d} \geq(1-\epsilon)$. Hence,

$$
\begin{aligned}
\underline{g}_{s, d}(A) & =\lim _{\mathcal{N} \ni N \rightarrow \infty} \frac{E_{s}\left(\omega_{N}^{*}\right)}{N^{1+s / d}} \geq(1-2 \rho)^{s} \liminf _{\mathcal{N} \ni N \rightarrow \infty} \frac{E_{s}\left(\omega_{N}^{K}\right)}{N^{1+s / d}} \\
& \geq(1-2 \rho)^{s} \liminf _{\mathcal{N} \ni N \rightarrow \infty} \frac{\mathcal{E}_{s}\left(K,\left|\omega_{N}^{2}\right|\right)}{\left|\omega_{N}^{2}\right|^{1+s / d}} \cdot\left(\frac{\left|\omega_{N}^{2}\right|}{N}\right)^{1+s / d} \\
& \geq(1-2 \rho)^{s}\left(1-k_{0} \rho^{s}-\chi_{0} \delta^{1 / 2}\right)^{1+s / d} \liminf _{N \rightarrow \infty} \frac{\mathcal{E}_{s}(K, N)}{N^{1+s / d}} \\
& \geq(1-\epsilon) \underline{g}_{s, d}(K)
\end{aligned}
$$

holds for any compact subset $K \subset A$ such that $\underline{\mathcal{M}}_{d}(K)>\mathcal{M}_{d}(A)-\delta$.

\section{Proofs of MAIN Theorems}

Proof of Theorem 1. First we remark that if $K \subset \mathbb{R}^{d}$ is compact, then $K$ is trivially a $d$-rectifiable manifold (or set) and so Theorem A shows, for $s>d$,

$$
g_{s, d}(K)=\frac{C_{s, d}}{\mathcal{L}_{d}(K)^{s / d}}
$$

Suppose $0<\epsilon<1$. Since $A \subset \mathbb{R}^{d^{\prime}}$ is a closed $d$-rectifiable set, Lemma 4 implies the existence of compact sets $K_{1}, K_{2}, K_{3}, \ldots \subset \mathbb{R}^{d}$ and bi-Lipschitz mappings $\psi_{i}: K_{i} \rightarrow \mathbb{R}^{d^{\prime}}, i=1,2,3, \ldots$, with constant $1+\epsilon$ such that $\psi_{1}\left(K_{1}\right), \psi_{2}\left(K_{2}\right)$,

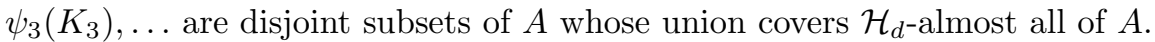

Let $n$ be large enough so that

$$
\mathcal{H}_{d}\left(\bigcup_{i=1}^{n} \psi_{i}\left(K_{i}\right)\right)=\sum_{i=1}^{n} \mathcal{H}_{d}\left(\psi_{i}\left(K_{i}\right)\right) \geq(1+\epsilon)^{-d} \mathcal{H}_{d}(A) .
$$


Since $\psi_{i}$ is bi-Lipschitz with constant $(1+\epsilon)$ we have

$$
\begin{aligned}
\bar{g}_{s, d}\left(\psi_{i}\left(K_{i}\right)\right) & \leq(1+\epsilon)^{s} g_{s, d}\left(K_{i}\right)=C_{s, d}(1+\epsilon)^{s} \mathcal{L}_{d}\left(K_{i}\right)^{-s / d} \\
& \leq C_{s, d}(1+\epsilon)^{2 s} \mathcal{H}_{d}\left(\psi_{i}\left(K_{i}\right)\right)^{-s / d}
\end{aligned}
$$

Applying Lemma 1 we obtain

$$
\begin{gathered}
\bar{g}_{s, d}(A) \leq \bar{g}_{s, d}\left(\bigcup_{i=1}^{n} \psi_{i}\left(K_{i}\right)\right) \leq\left(\sum_{i=1}^{n} \bar{g}_{s, d}\left(\psi_{i}\left(K_{i}\right)\right)^{-d / s}\right)^{-s / d} \\
\leq C_{s, d}(1+\epsilon)^{2 s}\left(\sum_{i=1}^{n} \mathcal{H}_{d}\left(\psi_{i}\left(K_{i}\right)\right)\right)^{-s / d} \leq C_{s, d}(1+\epsilon)^{3 s} \mathcal{H}_{d}(A)^{-s / d}
\end{gathered}
$$

We next provide a lower bound for $\underline{g}_{s, d}(A)$. Since $A$ is a closed $d$-rectifiable set, we have $\mathcal{M}_{d}(A)=\mathcal{H}_{d}(A)<\infty$ (cf. Lemma 3). Let $\delta>0$ be as in Lemma 5 , i.e., inequality (26) holds for every compact set $K \subset A$ such that $\mathcal{M}_{d}(K)>\mathcal{M}_{d}(A)-\delta$. Now let $n^{\prime}$ be large enough so that

$$
\mathcal{M}_{d}\left(\bigcup_{i=1}^{n^{\prime}} \psi_{i}\left(K_{i}\right)\right)=\sum_{i=1}^{n^{\prime}} \mathcal{H}_{d}\left[\psi_{i}\left(K_{i}\right)\right]>\mathcal{H}_{d}(A)-\delta=\mathcal{M}_{d}(A)-\delta .
$$

As in (34) we have

$$
\begin{aligned}
\underline{g}_{s, d}\left(\psi_{i}\left(K_{i}\right)\right) & \geq(1+\epsilon)^{-s} g_{s, d}\left(K_{i}\right)=C_{s, d}(1+\epsilon)^{-s} \mathcal{L}_{d}\left(K_{i}\right)^{-s / d} \\
& \geq C_{s, d}(1+\epsilon)^{-2 s} \mathcal{H}_{d}\left(\psi_{i}\left(K_{i}\right)\right)^{-s / d} .
\end{aligned}
$$

Then Lemma 2 and (36) give

$$
\begin{aligned}
\underline{g}_{s, d}(A) & \geq(1-\epsilon) \underline{g}_{s, d}\left(\bigcup_{i=1}^{n^{\prime}} \psi_{i}\left(K_{i}\right)\right) \geq(1-\epsilon)\left(\sum_{i=1}^{n^{\prime}} \underline{g}_{s, d}\left[\psi_{i}\left(K_{i}\right)\right]^{-d / s}\right)^{-s / d} \\
& \geq \frac{(1-\epsilon) C_{s, d}}{(1+\epsilon)^{2 s}}\left(\sum_{i=1}^{n^{\prime}} \mathcal{H}_{d}\left[\psi_{i}\left(K_{i}\right)\right]\right)^{-s / d} \geq \frac{(1-\epsilon) C_{s, d}}{(1+\epsilon)^{2 s}} \mathcal{H}_{d}(A)^{-s / d}
\end{aligned}
$$

Letting $\epsilon$ go to zero in (35) and (37), we obtain (11).

4.1. Proofs of Theorems $\mathbf{2}$ and 3. The following lemma relates the weighted minimal energy problem $(s \geq d)$ on a set $A \subset \mathbb{R}^{d^{\prime}}$ to the unweighted minimal energy problem on compact subsets of $A$. Theorems 2 and 3 then follow easily from this lemma.

For convenience, we define

$$
C_{d, d}:=\beta_{d}, \quad d \in \mathbb{N},
$$

where (cf. (3)) the constant $\beta_{d}$ is the volume of the unit ball in $\mathbb{R}^{d}$.

Lemma 6. Suppose $s \geq d, A \subset \mathbb{R}^{d^{\prime}}$ is compact with $\mathcal{H}_{d}(A)<\infty$, and that $w$ is a $C P D$-weight function on $A \times A$. Furthermore, suppose that for any compact subset $K \subset A$, the limit $g_{s, d}(K)$ exists and is given by

$$
g_{s, d}(K)=\frac{C_{s, d}}{\mathcal{H}_{d}(K)^{s / d}}
$$


Then

(a) $g_{s, d}^{w}(A)$ exists and is given by

$$
g_{s, d}^{w}(A)=C_{s, d}\left(\mathcal{H}_{d}^{s, w}(A)\right)^{-s / d}
$$

and,

(b) if a sequence $\left\{\widetilde{\omega}_{N}\right\}_{N=2}^{\infty}$, where $\widetilde{\omega}_{N}=\left\{x_{1}^{N}, \ldots, x_{N}^{N}\right\}$, is asymptotically $(w, s)$ energy minimizing on the set $A$ and $\mathcal{H}_{d}(A)>0$, then

$$
\frac{1}{N} \sum_{k=1}^{N} \delta_{x_{k}^{N}} \stackrel{*}{\longrightarrow} h_{d}^{s, w}, \quad N \rightarrow \infty,
$$

where $h_{d}^{s, w}$ is defined in (13).

Remark. If $\mathcal{H}_{d}(K)=0$, condition (38) is understood as $g_{s, d}(K)=\infty$.

Proof. To prove the first part of the theorem, we break $A$ into disjoint "pieces" of small diameter and estimate the $(w, s)$-energy of $A$ by replacing $w$ with its supremum or infimum on the resulting "pieces" and applying Lemmas 1 and 2.

For $\delta>0$, suppose that $\mathcal{P}_{\delta}$ is a partition of $A$ such that $\operatorname{diam} P \leq \delta$ and $\mathcal{H}_{d}(\bar{P})=\mathcal{H}_{d}(P)$ for $P \in \mathcal{P}_{\delta}$, where $\bar{B}$ denotes the closure of a set $B$. For each $P \in \mathcal{P}_{\delta}$, choose a closed subset $Q_{P} \subset P$ so that $\mathcal{Q}_{\delta}:=\left\{Q_{P}: P \in \mathcal{P}_{\delta}\right\}$ satisfies

$$
\sum_{P \in \mathcal{P}_{\delta}} \mathcal{H}_{d}\left(Q_{P}\right) \geq \mathcal{H}_{d}(A)-\delta
$$

An example of such systems $\mathcal{P}_{\delta}$ and $\mathcal{Q}_{\delta}$ can be constructed as follows. Let $G_{j}[t]$ be the hyperplane in $\mathbb{R}^{d^{\prime}}$ consisting of all points whose $j$-th coordinate equals $t$. If $(-a, a)^{d^{\prime}}$ is a cube containing $A$, then for $\mathbf{i}=\left(i_{1}, \ldots, i_{d^{\prime}}\right) \in\{1, \ldots, m\}^{d^{\prime}}$, let

$$
R_{\mathbf{i}}:=\left[t_{i_{1}-1}^{1}, t_{i_{1}}^{1}\right) \times \cdots \times\left[t_{i_{d^{\prime}}-1}^{d^{\prime}}, t_{i_{d^{\prime}}}^{d^{\prime}}\right),
$$

where $m$ and partitions $-a=t_{0}^{j}<t_{1}^{j}<\ldots<t_{m}^{j}=a, j=1, \ldots, d^{\prime}$, are chosen so that the diameter of every $R_{\mathbf{i}}, \mathbf{i} \in\{1, \ldots, m\}^{d^{\prime}}$, is less than $\delta$ and $\mathcal{H}_{d}\left(G_{j}\left[t_{i}^{j}\right] \cap A\right)=0$ for all $i$ and $j$. (Since $\mathcal{H}_{d}(A)<\infty$, there are at most countably many values of $t$ such that $\mathcal{H}_{d}\left(G_{j}[t] \cap A\right)>0$. $)$ Then, we may choose

$$
\mathcal{P}_{\delta}=\left\{R_{\mathbf{i}} \cap A: \mathbf{i} \in\{1, \ldots, m\}^{d^{\prime}}\right\}
$$

and $\gamma \in(0,1)$ sufficiently close to 1 such that $(41)$ holds for $\mathcal{Q}_{\delta}=\left\{Q_{\mathbf{i}}: \mathbf{i} \in\right.$ $\left.\{1, \ldots, m\}^{d^{\prime}}\right\}$, where $Q_{\mathbf{i}}=\left(\gamma\left(\bar{R}_{\mathbf{i}}-c_{\mathbf{i}}\right)+c_{\mathbf{i}}\right) \cap A$ and $c_{\mathbf{i}}$ denotes the center of $R_{\mathbf{i}}$.

For $B \subset A$, let

$$
\bar{w}_{B}=\sup _{x, y \in B} w(x, y) \text { and } \underline{w}_{B}=\inf _{x, y \in B} w(x, y)
$$

and define the simple functions

$$
\bar{w}_{\delta}(x):=\sum_{P \in \mathcal{P}_{\delta}} \bar{w}_{P} \cdot \chi_{P}(x) \text { and } \underline{w}_{\delta}(x):=\sum_{P \in \mathcal{P}_{\delta}} \underline{w}_{P} \cdot \chi_{P}(x),
$$


where $\chi_{K}$ denotes the characteristic function of a set $K$. Since the distance between any two sets from $\mathcal{Q}_{\delta}$ is strictly positive, Lemma 1 and equation (38) imply

$$
\begin{aligned}
& \bar{g}_{s, d}^{w}(A)^{-d / s} \geq \bar{g}_{s, d}^{w}\left(\bigcup_{Q \in \mathcal{Q}_{\delta}} Q\right)^{-d / s} \geq \sum_{\substack{Q \in \mathcal{Q}_{\delta} \\
Q \neq \emptyset}}\left(\bar{w}_{Q} \cdot \bar{g}_{s, d}(Q)\right)^{-d / s} \\
& =C_{s, d}^{-d / s} \sum_{\substack{Q \in \mathcal{Q}_{\delta} \\
Q \neq \emptyset}} \bar{w}_{Q}^{-d / s} \cdot \mathcal{H}_{d}(Q) \geq C_{s, d}^{-d / s} \int_{\substack{\cup Q \\
Q \in \mathcal{Q}_{\delta}}}\left(\bar{w}_{\delta}(x)\right)^{-d / s} d \mathcal{H}_{d}(x) .
\end{aligned}
$$

Applying Lemma 2 and relation (38), we similarly have

$$
\begin{gathered}
\underline{g}_{s, d}^{w}(A)^{-d / s} \leq \sum_{P \in \mathcal{P}_{\delta}}\left(\underline{w}_{P} \cdot \underline{g}_{s, d}(P)\right)^{-d / s}=\sum_{P \in \mathcal{P}_{\delta}}\left(\underline{w}_{P} \cdot \underline{g}_{s, d}(\bar{P})\right)^{-d / s} \\
=C_{s, d}^{-d / s} \sum_{P \in \mathcal{P}_{\delta}} \underline{w}_{P}^{-d / s} \cdot \mathcal{H}_{d}(\bar{P})=C_{s, d}^{-d / s} \int_{A}\left(\underline{w}_{\delta}(x)\right)^{-d / s} d \mathcal{H}_{d}(x) .
\end{gathered}
$$

Since $w$ is a CPD-weight function on $A \times A$, there is some neighborhood $G$ of $D(A)$ such that $\eta:=\inf _{G} w>0$. For $\delta>0$ sufficiently small, we have $P \times P \subset G$ for all $P \in \mathcal{P}_{\delta}$, and hence $\bar{w}_{\delta}(x) \geq w(x, x) \geq \underline{w}_{\delta}(x) \geq \eta$ for $x \in A$. Furthermore, $w$ is continuous at $(x, x) \in D(A)$ for $\mathcal{H}_{d}$-almost all $x \in A$ and thus, for any such $x$, it follows that $\bar{w}_{\delta}(x)$ and $\underline{w}_{\delta}(x)$ converge to $w(x, x)$ as $\delta \rightarrow 0$. Therefore, by the Lebesgue Dominated Convergence Theorem, the integrals

$$
\int_{\substack{\mathcal{U} Q \\ Q \in \mathcal{Q}_{\delta}}}\left(\bar{w}_{\delta}(x)\right)^{-d / s} d \mathcal{H}_{d}(x) \text { and } \int_{A}\left(\underline{w}_{\delta}(x)\right)^{-d / s} d \mathcal{H}_{d}(x)
$$

both converge to $\mathcal{H}_{d}^{s, w}(A)$ as $\delta \rightarrow 0$. Hence, using (42) and (43), we obtain (39).

To establish (40), suppose $\mathcal{H}_{d}(A)>0$ and that $\widetilde{\omega}_{N}=\left\{x_{1}^{N}, \ldots, x_{N}^{N}\right\}$ is an asymptotically $(w, s)$-energy minimizing sequence of $N$-point configurations on $A$. It is well-known $[18$, p. 9] that the weak* convergence result given in (40) is equivalent to the assertion that

$$
\lim _{N \rightarrow \infty} \frac{\left|\widetilde{\omega}_{N} \bigcap B\right|}{N}=h_{d}^{s, w}(B)
$$

holds for any almost clopen subset $B \subset A$, where we recall that a set $B \subset A$ is called almost clopen (with respect to $A$ and $\mathcal{H}_{d}$ ), if the $\mathcal{H}_{d}$-measure of the relative boundary of $B$ with respect to $A$ equals zero. Note that since $w$ is a CPD-weight, the relative boundary of $B$ also has $\mathcal{H}_{d}^{s, w}$-measure zero.

Let $B \subset A$ be almost clopen. Then $\bar{B}$ and $\overline{A \backslash B}$ satisfy the hypotheses of this lemma and hence also satisfy relation (39). Thus, for the asymptotically minimal sequence $\widetilde{\omega}_{N}$, we have

$$
\begin{aligned}
\lim _{N \rightarrow \infty} \frac{E_{s}^{w}\left(\widetilde{\omega}_{N}\right)}{\tau_{s, d}(N)} & =C_{s, d}\left(\mathcal{H}_{d}^{s, w}(A)\right)^{-s / d} \\
& =C_{s, d}\left(\mathcal{H}_{d}^{s, w}(\bar{B})+\mathcal{H}_{d}^{s, w}(\overline{A \backslash B})\right)^{-s / d} \\
& =\left(g_{s, d}^{w}(\bar{B})^{-d / s}+g_{s, d}^{w}(\overline{A \backslash B})^{-d / s}\right)^{-s / d} .
\end{aligned}
$$


Using relation (24) in Lemma 2 and (39) for $\bar{B}$ and $\overline{A \backslash B}$, we get

$$
\lim _{N \rightarrow \infty} \frac{\left|\widetilde{\omega}_{N} \bigcap B\right|}{N}=\frac{g_{s, d}^{w}(\overline{A \backslash B})^{d / s}}{g_{s, d}^{w}(\bar{B})^{d / s}+g_{s, d}^{w}(\overline{A \backslash B})^{d / s}}=h_{d}^{s, w}(B),
$$

where we used that $B$ is almost clopen. Thus (40) holds.

Theorems 2 and 3 then follow from Lemma 6 and Theorems 1 and B as we now explain. If $s>d$ and $A \subset \mathbb{R}^{d^{\prime}}$ is a closed $d$-rectifiable set, then every compact subset $B \subset A$ is also closed and $d$-rectifiable, and Theorem 1 implies that $B$ satisfies condition (38) and so Theorem 2 then follows from Lemma 6. If $s=d$ and $A$ is a compact subset of a $d$-dimensional $C^{1}$-manifold in $\mathbb{R}^{d^{\prime}}$, then applying Theorem $\mathrm{B}$ to every compact subset of $A$, we get (38). Consequently Theorem 3 follows from Lemma 6 with $s=d$.

\section{Proofs of the Separation Results: Theorem 4 and Corollary 1}

In this subsection we prove Theorem 4 and Corollary 1. For the proof of these results we will need Frostman's lemma establishing the existence of a non-trivial measure on $A$ satisfying a regularity assumption similar to the one in [19] for arclength.

Lemma 7 (see e.g. [17, Theorem 8.8]). Let $\alpha>0$ and $A$ be a Borel set in $\mathbb{R}^{d^{\prime}}$. Then $\mathcal{H}_{\alpha}(A)>0$ if and only if there is a Radon measure $\mu$ on $\mathbb{R}^{d^{\prime}}$ with compact support contained in $A$ such that $0<\mu(A)<\infty$ and

$$
\mu\left[B_{d^{\prime}}(x, r)\right] \leq r^{\alpha}, \quad x \in \mathbb{R}^{d^{\prime}}, \quad r>0 .
$$

Moreover, one can find $\mu$ so that $\mu(A) \geq c_{d^{\prime}, \alpha} \mathcal{H}_{\alpha}^{\infty}(A)$, where $c_{d^{\prime}, \alpha}>0$ is independent of $A$ and $\mathcal{H}_{\alpha}^{\infty}$ is given in (17).

We proceed with the proof of Theorem 4 using the technique developed in [16]. Let $\omega_{N}^{*}:=\left\{x_{1}, \ldots, x_{N}\right\}, N \in \mathbb{N}, N \geq 2$, be a $(w, s)$-energy minimizing configuration on $A$ (for convenience, we dropped the subscript $N$ in writing energy minimizing points $x_{k, N}$ ). For $i=1, \ldots, N$ let

$$
U_{i}(x):=\sum_{j \neq i} \frac{w\left(x, x_{j}\right)}{\left|x-x_{j}\right|^{s}}, \quad x \in A .
$$

From the minimization property we have that $U_{i}\left(x_{i}\right) \leq U_{i}(x), x \in A, i=1, \ldots, N$. If $\mu$ is a measure from Lemma 7 , set $r_{0}:=(\mu(A) / 2 N)^{1 / \alpha}$ and let

$$
D_{i}:=A \backslash \bigcup_{j \neq i} B_{d^{\prime}}\left(x_{j}, r_{0}\right), \quad i=1, \ldots, N
$$

Then, by the properties of $\mu$, we have

$$
\mu\left(D_{i}\right) \geq \mu(A)-\sum_{j \neq i} \mu\left[B_{d^{\prime}}\left(x_{j}, r_{0}\right)\right] \geq \mu(A)-(N-1) r_{0}^{\alpha}>\frac{\mu(A)}{2}>0,
$$


$i=1, \ldots, N$. Consequently,

$$
\begin{aligned}
U_{i}\left(x_{i}\right) & \leq \frac{1}{\mu\left(D_{i}\right)} \int_{D_{i}} U_{i}(x) d \mu(x) \leq \frac{2}{\mu(A)} \sum_{j \neq i} \int_{D_{i}} \frac{w\left(x, x_{j}\right)}{\left|x-x_{j}\right|^{s}} d \mu(x) \\
& \leq \frac{2\|w\|}{\mu(A)} \sum_{j \neq i} \int_{A \backslash B_{d^{\prime}}\left(x_{j}, r_{0}\right)} \frac{1}{\left|x-x_{j}\right|^{s}} d \mu(x), \quad i=1, \ldots, N,
\end{aligned}
$$

where $\|w\|:=\sup \{|w(x, y)|: x, y \in A\}$. Let $R:=\operatorname{diam} A$. Then by (45) we have $\mu(A) \leq R^{\alpha}$. For every $y \in A$ and $r \in(0, R]$, using (45) we also get

$$
\begin{aligned}
T_{s}(y, r) & :=\int_{A \backslash B_{d^{\prime}}(y, r)} \frac{1}{|x-y|^{s}} d \mu(x) \leq \int_{0}^{r^{-s}} \mu\left\{x \in A: \frac{1}{|x-y|^{s}}>t\right\} d t \\
& =\frac{\mu(A)}{R^{s}}+\int_{R^{-s}}^{r^{-s}} \mu\left[B_{d^{\prime}}\left(y, t^{-1 / s}\right)\right] d t \leq R^{\alpha-s}+\int_{R^{-s}}^{r^{-s}} t^{-\alpha / s} d t \\
& \leq \begin{cases}\frac{s}{(s-\alpha)} r^{\alpha-s}, & s>\alpha, \\
1+\alpha \ln \frac{R}{r}, & s=\alpha .\end{cases}
\end{aligned}
$$

Then for $i=1, \ldots, N$ and $s>\alpha$ we have

$$
U_{i}\left(x_{i}\right) \leq \frac{2\|w\|}{\mu(A)} \sum_{j \neq i} T_{s}\left(x_{j}, r_{0}\right) \leq \frac{2 s(N-1)\|w\|}{(s-\alpha) \mu(A) r_{0}^{s-\alpha}} \leq C_{1}\|w\|\left(\frac{N}{\mu(A)}\right)^{s / \alpha},
$$

where $C_{1}>0$ is a constant independent of $A, w$ and $N$. Hence,

$$
\mathcal{E}_{s}^{w}(A, N)=E_{s}^{w}\left(\omega_{N}^{*}\right)=\sum_{i=1}^{N} U_{i}\left(x_{i}\right) \leq \frac{M_{s, \alpha}\|w\|}{\mathcal{H}_{\alpha}^{\infty}(A)^{s / \alpha}} N^{1+s / \alpha},
$$

where $M_{s, \alpha}$ is a constant independent of $A, w$, and $N$ which establishes Corollary 1 in the case $s>\alpha$. In particular, the above estimates show that we can take

$$
M_{s, \alpha}=\frac{s 2^{s / \alpha}}{(s-\alpha)\left(c_{d^{\prime}, \alpha}\right)^{s / \alpha}} .
$$

Since $w$ is a CPD-weight function, there are constants $\eta, \rho>0$ such that $w(x, y)>\eta$ whenever $|x-y|<\rho$. Assume that $\delta\left(\omega_{N}^{*}\right)<\rho$ and let $i_{s}$ and $j_{s}$ be such that $\delta\left(\omega_{N}^{*}\right)=\left|x_{i_{s}}-x_{j_{s}}\right|$. Then with some constant $C_{2}>0$ independent of $N$ and the choice of $\omega_{N}^{*}$ we obtain from (46)

$$
C_{2} N^{s / \alpha} \geq U_{i_{s}}\left(x_{i_{s}}\right) \geq \frac{w\left(x_{i_{s}}, x_{j_{s}}\right)}{\left|x_{i_{s}}-x_{j_{s}}\right|^{s}} \geq \frac{\eta}{\left|x_{i_{s}}-x_{j_{s}}\right|^{s}}=\frac{\eta}{\delta\left(\omega_{N}^{*}\right)^{s}} .
$$

Hence,

$$
\delta\left(\omega_{N}^{*}\right) \geq C_{0} N^{-1 / \alpha}
$$

where $C_{0}=C_{0}(A, w, \alpha, s)>0$. Thus, in any case,

$$
\delta\left(\omega_{N}^{*}\right) \geq \min \left\{\rho, C_{0} N^{-1 / \alpha}\right\} \geq C_{s} N^{-1 / \alpha}, \quad N \geq 2,
$$

for a sufficiently small constant $C_{s}>0$ independent of $N$ and $\omega_{N}^{*}$. In particular, when $w \equiv 1$, we have

$$
\delta\left(\omega_{N}^{*}\right) \geq \frac{c_{s, \alpha}}{\left(\mathcal{H}_{\alpha}^{\infty}(A) \cdot N\right)^{1 / \alpha}}
$$


The case $s=\alpha$ is handled analogously, which completes the proofs of Theorem 4 and Corollary 1.

\section{Proof of Theorem 5}

The essential ingredient in the proof of Theorem 5 is the following lemma which assumes lower regularity. Consistent with the definition in (20), we say that a set $K \subset \mathbb{R}^{d^{\prime}}$ is lower $\alpha$-regular if there are positive constants $C_{0}$ and $r_{0}$ so that

$$
\left(C_{0}\right)^{-1} r^{\alpha} \leq \mathcal{H}_{\alpha}\left(K \cap B_{d^{\prime}}(x, r)\right)
$$

for all $x \in K$ and $r<r_{0}$.

Lemma 8. Suppose $K \subset \mathbb{R}^{d^{\prime}}$ is compact and lower $\alpha$-regular and $a \in K$. Further suppose $s>\alpha$ and $w: K \times K \rightarrow[0, \infty]$ is a CPD-weight function on $K^{\prime} \times K^{\prime}$ for any compact $K^{\prime} \subset K \backslash\{a\}$. If $w$ has a zero of order at most $t$ at $(a, a)$, where $0<t<s$, then

$$
\underline{g}_{s, \alpha}^{w}(K) \geq C_{1} C_{0}^{-s / \alpha} 2^{-(s+t)}\left(\int_{K} \frac{1}{|x-a|^{(t \alpha) / s}} d \mathcal{H}_{\alpha}(x)\right)^{-s / \alpha} .
$$

Proof. Let $\omega_{N}=\left\{x_{1}, \ldots, x_{N}\right\}$ be a configuration of $N$ distinct points in $K$. For $i=1, \ldots, N$, let $\rho_{i}=\left|x_{i}-a\right|, r_{i}=\min _{j \neq i}\left|x_{i}-x_{j}\right|$, and choose $y_{i} \in \omega_{N}$ such that $\left|x_{i}-y_{i}\right|=r_{i}$. Since $K$ is bounded, there is some finite $L$ (independent of $N$ ) such that there are at most $L-1$ of the points $x_{i} \in \omega_{N}$ with the property that $r_{i} \geq r_{0}$. We order the points in $\omega_{N}$ so that $\rho_{N} \leq \rho_{i}$ for $i=1, \ldots, N$ and so that $r_{i}<r_{0}$ for $i=1, \ldots, N-L$. It follows from Cauchy's and Jensen's inequality (see (29) of [12]) that if $\gamma_{1}, \ldots, \gamma_{M}$ are positive numbers, then

$$
\sum_{i=1}^{M} \gamma_{i}^{-s} \geq M^{1+s / \alpha}\left(\sum_{i=1}^{M} \gamma_{i}^{\alpha}\right)^{-s / \alpha}
$$

from which we obtain

$$
\begin{aligned}
E_{s}^{w}\left(\omega_{N}\right) & \geq \sum_{i=1}^{N-L} \frac{w\left(x_{i}, y_{i}\right)}{r_{i}^{s}} \geq C_{1} \sum_{i=1}^{N-L} \frac{\rho_{i}^{t}}{r_{i}^{s}} \\
& \geq C_{1}(N-L)^{1+s / \alpha}\left(\sum_{i=1}^{N-L} \frac{r_{i}^{\alpha}}{\rho_{i}^{t \alpha / s}}\right)^{-s / \alpha} .
\end{aligned}
$$

For $i=1, \ldots, N-1$, observe that

$$
r_{i}=\min _{j \neq i}\left|x_{i}-x_{j}\right| \leq\left|x_{i}-a\right|+\min _{j \neq i}\left|a-x_{j}\right| \leq \rho_{i}+\rho_{N} \leq 2 \rho_{i}
$$

and so, for $x \in B_{d^{\prime}}\left(x_{i}, r_{i} / 2\right)$,

$$
|x-a| \leq\left|x-x_{i}\right|+\left|x_{i}-a\right| \leq r_{i} / 2+\rho_{i} \leq 2 \rho_{i} .
$$

Using (47) and (51) we have

$$
\frac{r_{i}^{\alpha}}{\rho_{i}^{t \alpha / s}} \leq C_{0} 2^{(\alpha / s)(s+t)} \int_{K \cap B_{d^{\prime}}\left(x_{i}, r_{i} / 2\right)} \frac{1}{|x-a|^{t \alpha / s}} d \mathcal{H}_{\alpha}(x)
$$


for $i=1, \ldots, N-L$. Since $B_{d^{\prime}}\left(x_{i}, r_{i} / 2\right)$ and $B_{d^{\prime}}\left(x_{j}, r_{j} / 2\right)$ are disjoint for $i \neq j$, it follows that

$$
\sum_{i=1}^{N-L} \frac{r_{i}^{\alpha}}{\rho_{i}^{t \alpha / s}} \leq C_{0} 2^{(\alpha / s)(s+t)} \int_{K} \frac{1}{|x-a|^{t \alpha / s}} d \mathcal{H}_{\alpha}(x),
$$

which combined with (50) completes the proof.

Remark. If $K$ is $\alpha$-regular (as opposed to only lower $\alpha$-regular) at $a$ in the above lemma, then the integral $\int_{K \cap B_{d^{\prime}}(a, \delta)} \frac{1}{|x-a|^{(t \alpha) / s}} d \mathcal{H}_{\alpha}(x)$ appearing in (48) is finite for $\delta$ sufficiently small (cf. [17, p. 109]) and thus the Lebesgue Dominated Convergence Theorem gives

$$
\lim _{\delta \rightarrow 0} \int_{K \cap B_{d^{\prime}}(a, \delta)} \frac{1}{|x-a|^{(t \alpha) / s}} d \mathcal{H}_{\alpha}(x)=0
$$

and so $\lim _{\delta \rightarrow 0} g_{s, \alpha}^{w}\left(K \cap B_{d^{\prime}}(a, \delta)\right)=\infty$.

Now we are prepared to complete the proof of Theorem 5. First note that the hypotheses of Theorem 5 (namely that $A$ is $\alpha_{i}$-regular at $a_{i}$ and $w$ has a zero of order of at most $t<s$ at $a_{i}$ for $\left.i=1, \ldots, n\right)$ imply that $\int_{A} w(x, x)^{-d / s} d \mathcal{H}_{d}(x)<\infty$.

Suppose $\epsilon>0$. By Lemma 8 and Lemma 2 we can find $\delta>0$ such that $B_{\epsilon}:=$ $\bigcup_{i=1}^{n}\left(A \cap B_{d^{\prime}}\left(a_{i}, \delta\right)\right)$ satisfies $\underline{g}_{s, d}^{w}\left(B_{\epsilon}\right) \geq \epsilon^{-1}$ (note that if $\alpha<d$ and $\underline{g}_{s, \alpha}^{w}(K)>0$, then $\left.\underline{g}_{s, d}^{w}(K)=\infty\right)$ and $\mathcal{H}_{d}^{s, w}\left(A_{\epsilon}\right)=\int_{A_{\epsilon}} w(x, x)^{-d / s} d \mathcal{H}_{d}(x) \geq(1-\epsilon) \mathcal{H}_{d}^{s, w}(A)$, where $A_{\epsilon}:=A \backslash B_{\epsilon}$.

Since $w$ is a CPD-weight function on $A_{\epsilon} \times A_{\epsilon}$, it follows from Theorem 2 that $g_{s, d}^{w}\left(A_{\epsilon}\right)$ exists and equals $C_{s, d} \mathcal{H}_{d}^{s, w}\left(A_{\epsilon}\right)^{-s / d}$. Lemma 2 then gives

$$
\begin{aligned}
\underline{g}_{s, d}^{w}(A) & \geq\left(g_{s, d}^{w}\left(A_{\epsilon}\right)^{-d / s}+\underline{g}_{s, d}^{w}\left(B_{\epsilon}\right)^{-d / s}\right)^{-s / d} \\
& \geq\left(C_{s, d}^{-d / s} \mathcal{H}_{d}^{s, w}\left(A_{\epsilon}\right)+\epsilon^{d / s}\right)^{-s / d} \\
& \geq\left(C_{s, d}^{-d / s} \mathcal{H}_{d}^{s, w}(A)+\epsilon^{d / s}\right)^{-s / d} .
\end{aligned}
$$

Also, we clearly have

$$
\bar{g}_{s, d}^{w}(A) \leq g_{s, d}^{w}\left(A_{\epsilon}\right)=C_{s, d} \mathcal{H}_{d}^{s, w}\left(A_{\epsilon}\right)^{-s / d} \leq C_{s, d}(1-\epsilon)^{-s / d} \mathcal{H}_{d}^{s, w}(A)^{-s / d} .
$$

Taking $\epsilon \rightarrow 0$ in $(52)$ and (53) shows that $g_{s, d}^{w}(A)$ exists and equals $C_{s, d} \mathcal{H}_{d}^{s, w}(A)^{-s / d}$. If $\mathcal{H}_{d}^{s, w}(A)>0$, then, as in the proof of Theorem 2, Lemma 2 implies that $(15)$ holds for any asymptotically $(w, s)$-energy minimizing sequence of configurations $\widetilde{\omega}_{N}=\left\{x_{1}^{N}, \ldots, x_{N}^{N}\right\}, N=2,3, \ldots$, for $A$ which completes the proof of Theorem 5 .

\section{ACKNOWLEDGEMENT}

We thank the anonymous referee for a careful reading of this manuscript and for helpful comments and suggestions.

\section{REFERENCES}

[1] N.N. Andreev, An extremal property of the icosahedron, East J. Approx. 2 (1996), no. 4, 459-462. MR1426716 (97m:52022)

[2] N.N. Andreev, On positions of points with minimal energy, Papers of the V.A. Steklov Math. Institute. 219 (1997), 27-31. MR1642295 (99i:52019)

[3] J. Benedetto, M. Fickus, Finite normalized tight frames, Adv. Comput. Math. 18 (2003), 357-385. MR1968126 (2004c:42059) 
[4] S.V. Borodachov, On minimization of the energy of varying range interactions on one- and multidimensional conductors, Ph.D. Thesis, Vanderbilt University (2006).

[5] M. Bowick, D.R. Nelson, A. Travesset, Interacting topological defects in frozen topographies, Phys. Rev. B 62 (2000), 8738-8751.

[6] J.H. Conway, N.J.A. Sloane, Sphere Packings, Lattices and Groups, Springer Verlag, New York: 3rd ed., 1999. MR1662447 (2000b:11077)

[7] B.E.J. Dahlberg, On the distribution of Fekete points, Duke Math. J. 45 (1978), 537-542. MR507457 (80c:31003)

[8] S. Damelin, V. Maymeskul, On mesh norm of s-extremal configurations on compact sets in $\mathbb{R}^{n}$, Constructive Functions Tech-04, 2004 (Atlanta, GA).

[9] P.D. Dragnev, D.A. Legg, D.W. Townsend, Discrete logarithmic energy on the sphere, Pacific J. Math. 207 (2002), no. 2, 345-358. MR1972249 (2004c:52015)

[10] H. Federer, Geometric measure theory, Springer-Verlag, Berlin-Heidelberg-New York, 1969, 680 pages. MR0257325 (41:1976)

[11] L. Fejes Toth, Regular Figures, Pergamon Press, Berlin-Göttingen-Heidelberg, 1953.

[12] D.P. Hardin, E.B. Saff, Minimal Riesz energy point configurations for rectifiable $d$-dimensional manifolds, Adv. Math. 193 (2005), 174-204. MR2132763 (2005m:49006)

[13] D.P. Hardin, E.B. Saff, Discretizing manifolds via minimum energy points, Notices Amer. Math. Soc. 51 (2004), no. 10, 1186-1194. MR2104914 (2006a:41049)

[14] A.V. Kolushov, V.A. Yudin, On the Korkin-Zolotarev construction, Discrete Mathematics 6 (1994), no. 1, 155-157 (in Russian). MR1273240 (95j:52033)

[15] A.V. Kolushov, V.A. Yudin, Extremal depositions of points on the sphere, Anal. Math. 23 (1997), no. 1, 25-34. MR1630001 (99f:41039)

[16] A.B.J. Kuijlaars, E.B. Saff, Asymptotics for minimal discrete energy on the sphere, Trans. Amer. Math. Soc. 350 (1998), no. 2, 523-538. MR1458327 (98e:11092)

[17] P. Mattila, Geometry of sets and measures in Eucledian spaces. Fractals and Rectifiability, Cambridge Univ. Press, 1995, 344 pages. MR1333890 (96h:28006)

[18] N.S. Landkof, Foundations of modern potential theory. Springer-Verlag, Berlin-HeidelberNew York, 1972, 426 pages. MR0350027 (50:2520)

[19] A. Martinez-Finkelstein, V. Maymeskul, E.A. Rakhmanov, E.B. Saff, Asymptotics for minimal discrete Riesz energy on curves in $\mathbb{R}^{d}$ Canad. Math. J., 56 (2004), 529-552. MR2057285 (2005a:31010)

[20] T.W. Melnyk, O. Knop, and W. R. Smith, Extremal Arrangements of Points and Unit Charges on a Sphere: Equilibrium Configurations Revisited, Canad. J. Chem. 55, (1977), 1745-1761. MR0444497 (56:2848)

[21] I.H. Sloan, R.S. Womersley, Extremal systems of points and numerical integration on the Sphere, Adv. Comp. Math. 21 (2004), 102-125. MR2065291 (2005b:65024)

[22] S. Smale, Mathematical problems for the next century, Mathematical Intelligencer, 20 (1998), 7-15. MR1631413 (99h:01033)

[23] V.A. Yudin, The minimum of potential energy of a system of point charges, Discrete Math. Appl. 3 (1993), no. 1, 75-81. MR1181534 (93f:31008)

Department of Mathematics, Center for Constructive Approximation, Vanderbilt

University, NAshville, Tennessee 37240

E-mail address: sergiy.v.borodachov@vanderbilt.edu

Current address: School of Mathematics, Georgia Institute of Technology, Atlanta, Georgia 30332

E-mail address: borodasv@math.gatech.edu

Department of Mathematics, Center for Constructive Approximation, Vanderbilt

University, NAshville, Tennessee 37240

E-mail address: doug.hardin@vanderbilt.edu

Department of Mathematics, Center for Constructive Approximation, Vanderbilt University, Nashville, Tennessee 37240

E-mail address: edward.b.saff@vanderbilt.edu 